\title{
STABILITY OF IRSL SIGNALS FROM SEDIMENTARY K-FELDSPAR SAMPLES
}

\author{
KRISTINA J. THOMSEN ${ }^{1}$, ANDREW S. MURRAY ${ }^{2}$ and MAYANK JAIN ${ }^{1}$ \\ ${ }^{I}$ Radiation Research Division, Risø National Laboratory for Sustainable Energy, Technical University of Denmark, \\ DK-4000 Roskilde, Denmark \\ ${ }^{2}$ Nordic Laboratory for Luminescence Dating, Department of Earth Sciences, Aarhus University, Risø DTU, \\ DK-4000 Roskilde, Denmark
}

Received 15 June 2010

Accepted 29 September 2010

\begin{abstract}
Recent work has identified IR stimulated luminescence signals at elevated temperature from both potassium- and sodium-rich feldspars that have much lower anomalous fading rates than the conventional signal measured using IR stimulation at $50^{\circ} \mathrm{C}$. This paper examines the stability of these signals for potassium-rich sedimentary feldspars. We show that the natural post-IR IRSL (pIRIR) signal from a 3.6 Ma old sample is in apparent saturation on a laboratory generated dose response curve, i.e. it does not show detectable fading in nature although a low fading rate is observed on laboratory time scales. We show that the pIRIR signal has a greater thermal stability than the IRSL signal and that the trend in increasing thermal stability is mirrored by a decreasing fading rate. We also investigate the effect of preheat temperature and IR stimulation power on the decay shape and conclude that the data can be explained in terms of either a single- or multiple-trap model. We present evidence that may suggest that at least part of pIRIR signal is derived from a high temperature trap $\left(\sim 550^{\circ} \mathrm{C}\right.$ thermoluminescence (TL) peak), although again the data can also be explained in terms of a single-trap model. Finally, we present dose response curves and characteristic curvature constants $\left(D_{0}\right)$ values for various IRSL signals and conclude that the more stable signals saturate more quickly than the less stable signals and that the initial and final signals saturate at approximately the same level.
\end{abstract}

Keywords: K-feldspar, Anomalous fading, IRSL, thermal stability, post-IR IRSL.

\section{INTRODUCTION}

In retrospective dose determinations using optically stimulated luminescence (OSL), quartz is often the preferred dosimeter because, in most environments, it has proved to be both sensitive and accurate. However, one major drawback is that the most light-sensitive part of the quartz OSL signal (the so-called fast component) generally saturates at $<200 \mathrm{~Gy}$, which usually limits the applicable age range to $<150 \mathrm{ka}$. In contrast, OSL signals from

Corresponding author: K. J. Thomsen e-mail: krth@risoe.dtu.dk feldspars (using infrared (IR) stimulation at $50^{\circ} \mathrm{C}$ ) continue to grow to much higher doses $(>1 \mathrm{kGy})$ and thus making feldspar a strong candidate for extending the datable OSL age range. Unfortunately feldspars suffer from one major drawback, anomalous (athermal) fading (Wintle, 1973, Spooner, 1994) in which the OSL signal decreases with storage time faster than expected from its kinetic characteristics. Procedures for correcting for this instability exist, but such corrections can be large $(>30 \%)$ and only apply to the linear (low dose) part of the dose response curve (e.g. Huntley and Lamothe, 2001, Lamothe et al., 2003). Thus, identification of a signal 
much less prone to anomalous fading than the IRSL signal measured at $50^{\circ} \mathrm{C}(\operatorname{IR}(50))$ is very desirable, if the datable age range is to be extended using feldspar.

Thomsen et al. (2008) investigated the laboratory fading rates of various luminescence signals from feldspar sediment extracts and found that the fading rate decreased as a function of both stimulation time and increasing stimulation temperature. They also investigated the characteristics of the IRSL signal measured at elevated temperature following an IR stimulation at some lower temperature, in their case $50^{\circ} \mathrm{C}$; they termed this a post-IR IR (pIRIR) signal. The lowest fading rate in their study was observed for the pIRIR $(50,225)$ signal (the two numbers in brackets give the stimulation temperature of the first and second IR stimulation, respectively) using the later part of the signal collected between $\sim 30 \mathrm{~s}$ and $\sim 70 \mathrm{~s}$ of stimulation with an IR power density of $\sim 135 \mathrm{~mW} / \mathrm{cm}^{2}$. Fig. 1 summarises their observations most relevant to this paper (based on their table 3). For their K-rich feldspar extracts from glaciofluvial sediment samples, the average fading rates for the IR(50) signal were $\sim 3 \%$ /decade for the initial $1.15 \mathrm{~s}$ of the signal. If the stimulation temperature was increased to $225^{\circ} \mathrm{C}$, the average fading rate dropped to $\sim 2 \% /$ decade, and for the post-IR IR signal the laboratory fading rate decreased further, to just over $1 \% /$ decade. The fading rates for the 30 to $70 \mathrm{~s}$ signal (less a background derived from the last $19.2 \mathrm{~s}$ of the $100 \mathrm{~s}$ stimulation period) were consistently lower, starting at just under $1.5 \% /$ decade for the IR(50) signal, and decreasing to $\sim 0.6 \% /$ decade for the pIRIR $(50,225)$ signal.

The fading rate observations of Thomsen et al. (2008) were all concerned with the behaviour of laboratory induced signals. Buylaert et al. (2009) examined whether

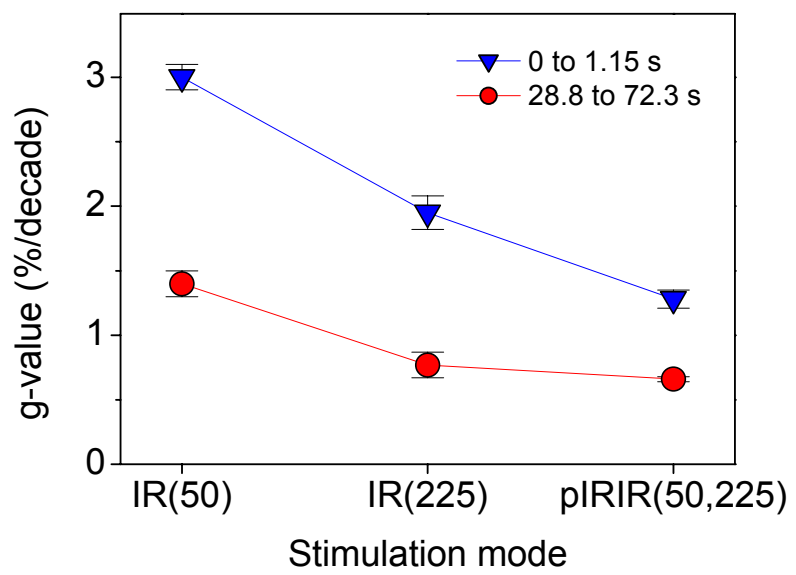

Fig. 1. Average fading rates (\%/decade; $t_{c}=2$ days) for IR stimulation using a preheat of $250^{\circ} \mathrm{C}$ for $60 \mathrm{~s}$ and $100 \mathrm{~s}$ of IR stimulation (data taken from table 3 in Thomsen et al., 2008). For IR stimulation at $50^{\circ} \mathrm{C}$ these data are from samples 963806, 951012, 951020 and 970204. For IR stimulation at $225^{\circ} \mathrm{C}$ the values are averages of the results obtained from samples 963806, 951012, 951020, 970204 and 972516. For pIRIR $(50,225)$ stimulation the values are averages of the results obtained from samples 963806, 951012 and 951020. these conclusions also applied to signals induced by irradiation in nature, by testing the dating potential of the initial pIRIR $(50,225)$ signal following a preheat at $250^{\circ} \mathrm{C}$ for $60 \mathrm{~s}$ from K-rich feldspar extracts from a variety of sedimentary samples; they concluded that the fadingcorrected ages obtained using the $\operatorname{IR}(50)$ and the pIRIR $(50,225)$ signals were consistent with each other, but the fading correction applied to the pIRIR $(50,225)$ signal was on average $\sim 39 \%$ lower than that applied to the IR(50) signal. This suggested that the lower laboratory fading rates observed for the pIRIR $(50,225)$ signal in the laboratory provided an appropriate measure of the fading rates in nature. Buylaert et al. (2009) did not attempt to use the later part of the signal for age determination.

Thiel et al. (2010) applied a pIRIR(50, 290) protocol applying a preheat of $320^{\circ} \mathrm{C}$ for $60 \mathrm{~s}$ to their polymineral fine grain samples (i.e. giving a signal derived from a mixture of feldspars), and found for the oldest samples (with an expected dose of $\sim 2.4 \mathrm{kGy}$ ) that the natural signal was in, or close to, saturation. This important observation suggests that the fading of this signal was negligible in nature. However, Thiel et al. (2010) also measured laboratory fading rates of $1-1.5 \% /$ decade on this and similar samples, which is inconsistent with the natural signal being in laboratory saturation.

Thomsen et al. (2008) observed that the reduction in dose (measured using the IR(50) signal) as a function of prior UV exposure (using a solar simulator; Hönle Sol 2) could be represented well by a single exponential decay, and thus concluded that a single trap was being emptied. Murray et al. (2009) argued that for their samples the main source of the IR(50) signal was probably an IR bleachable TL peak at $\sim 410^{\circ} \mathrm{C}$; this observation supports the suggestion that the IRSL is derived from a single trap.

Thomsen et al. (2008) explained their observations using the models for luminescence production in feldspars originally proposed and developed by Poolton et al. (1994, 1995, 2002a,b), in which the IR(50) excites the trapped electrons to an excited state from where they are able to tunnel to nearby recombination centres. In this model, by increasing the IR stimulation temperature some trapped electrons are given sufficient thermal energy to be stimulated into band-tail states; thus electrons from stable traps (with no nearby recombination centres) are able to migrate to more and more distant recombination centres to give an IRSL signal. Based on this model, the pIRIR signal is expected to be more stable than the IR(50) signal because the initial IR(50) stimulation results in the recombination of all closely-spaced donoracceptor pairs, leaving behind only those electrons in traps which are distant from a populated recombination site and thus unlikely to have undergone anomalous fading in nature.

This paper investigates in more detail the origins of the pIRIR signal and compares them to the origin of the standard IRSL signal measured at $50^{\circ} \mathrm{C}$. In particular we 
examine whether the two signals originate with the same electron trap or with two different electron traps. In section 3 we look at evidence for the pIRIR signal being more stable than the IR(50) signal. In section 4 we present pulse anneal data to investigate the thermal stability of various IRSL signals and compare these with g-values of the respective signals. In section 5 we investigate whether the shape of the IRSL decay curve contains information on the origins of the signal by examining the effect of preheat temperature and stimulation power on decay shape. In section 6 we investigate whether a high temperature TL peak (centred at $\sim 550^{\circ} \mathrm{C}$ ) is the source of the elevated temperature IRSL signal. Finally in section 7 we present dose response curves and characteristic curvature constants $\left(\mathrm{D}_{0}\right)$ values for various IRSL signals to establish if these signals are dosimetrically useful.

\section{EXPERIMENTAL DETAILS}

\section{Samples}

In this study five different coarse-grain sedimentary K-feldspar samples have been measured (lab codes: 963806, 951012, 951020, 981014 and 062213). The sediments were first wet-sieved and treated with $\mathrm{HCl}$ and $\mathrm{H}_{2} \mathrm{O}_{2}$ to remove carbonates and organic material, and then density separated $\left(\rho<2.58 \mathrm{~g} / \mathrm{cm}^{3}\right)$ using heavy liquids. The lighter (K-rich feldspar) fractions were etched for $40 \mathrm{~min}$ in $10 \% \mathrm{HF}$ to remove outer layers. After etching, any contaminating fluorides were dissolved using $\mathrm{HCl}$, before final rinsing and drying.

Sample 963906 from Russia $(106-212 \mu \mathrm{m})$, and $951012(106-212 \mu \mathrm{m})$ and $951020(212-300 \mu \mathrm{m})$ from Greenland, are all of glaciofluvial origin. 981014 from Denmark $(90-150 \mu \mathrm{m})$ is a shallow marine Eemian sample; further information can be found in Buylaert et al. (2010). Sample 062213 was collected at the base of the Vale de Freixo Pliocene succession, in central Portugal. The sampled fossiliferous silty fine sand bed, in which the identification of a calcareous nannofossil assemblage, marine mollusks and isotopic Sr dating of shells, indicates an age of $\sim 3.6 \mathrm{Ma}$ (Ramos and Cunha, 2004; Silva et al., 2010).

\section{Instrumentation}

Automated Risø TL/OSL readers, model Risø TL/OSL-DA-15 and 20, were used for the OSL measurements. Infrared stimulation used an IR $(870 \mathrm{~nm}$, FWHM $40 \mathrm{~nm}$ ) LED array delivering approximately 135 $\mathrm{mW} / \mathrm{cm}^{2}$ at the sample (Bøtter-Jensen et al., 2003). Detection was in the blue region of the spectrum, through a combination of Corning 7-59 and BG-39 glass filters. All heating rates were $5^{\circ} \mathrm{C} / \mathrm{s}$ in a nitrogen atmosphere except for the thermoluminescence experiments (section 6) in which a heating rate of $1{ }^{\circ} \mathrm{C} / \mathrm{s}$ was employed. Laboratory irradiations used calibrated ${ }^{90} \mathrm{Sr} /{ }^{90} \mathrm{Y}$ beta sources mounted on the readers (quartz dose rates ranged between 0.14
Gy/s and $0.18 \mathrm{~Gy} / \mathrm{s})$. Samples were mounted in stainless steel cups using silicone oil.

\section{SIGNALS FROM A SAMPLE WITH THE NATURAL SIGNAL IN SATURATION}

Murray et al. (2009) showed that for their samples reliable dose estimates for the IR(50) signal could be derived using preheat temperatures up to $320^{\circ} \mathrm{C}$. This observation is important because it suggests that one can use preheat temperatures at least up to $\sim 320^{\circ} \mathrm{C}$ without significant emptying of the dosimetry trap(s). This implies that we can use stimulation temperatures up to $\sim 300^{\circ} \mathrm{C}$. Using blue (7-59 and BG-39) filters, Thiel et al. (2010) observed a pIRIR $(50,290)$ signal from a natural sample preheated to $320^{\circ} \mathrm{C}$ for $60 \mathrm{~s}$ that appeared to be at, or close to, the saturation light level observed in a dose response curve regenerated in the laboratory. This observation made use of polymineral fine grains $(4-11 \mu \mathrm{m})$ from an 800 ka European loess sample expected to have absorbed a natural dose of $\sim 2.4 \mathrm{kGy}$ since last exposure to light. Although it is very likely that this signal originated from a mixture of different feldspar grains, and so the dominant mineral source is poorly known, the clear implication of this result is that this pIRIR signal does not show any detectable loss in nature over a period of $\sim 800 \mathrm{ka}$, either because of thermal instability, or because of anomalous fading. To test whether this important observation also applies to coarse-grained K-rich feldspar extracts; we have repeated the experiment using $180-250 \mathrm{~mm}$ grains extracted from a Pliocene marine level dated to be $\sim 3.6 \mathrm{Ma}$ old (sample 062213) and thus expected to have absorbed a natural dose of $\sim 10 \mathrm{kGy}$, which is well beyond the dose at which saturation occurs for feldspar. Thus, in the absence of significant anomalous fading we would expect to measure a natural signal that is at the saturation level of the dose response curve. The SAR sequence employed here used a preheat of $320^{\circ} \mathrm{C}$ for $60 \mathrm{~s}$, and a first IR stimulation at $50^{\circ} \mathrm{C}$ for $100 \mathrm{~s}$ followed immediately by a $2^{\text {nd }}$ IR stimulation at $290^{\circ} \mathrm{C}$ for $400 \mathrm{~s}$. Typical decay curves $(0-100 \mathrm{~s})$ resulting from the 400 Gy test dose are shown inset to Fig. 2a $(\operatorname{IR}(50))$ and $\mathbf{2 b}\left(\operatorname{pIRIR}\left(50,290^{\circ} \mathrm{C}\right)\right)$. The intensity of the pIRIR $(50,290)$ signal is $\sim 5$ times greater than that of the IR(50) signal, Fig. 2a shows the dose response for a single aliquot of the sensitivity corrected $50^{\circ} \mathrm{C}$ IRSL, both for the initial (0-1.44 s) part of the decay curve (circles) and for the signal observed further down the curve (50-60 s, triangles). In both cases a background based on the last $15.2 \mathrm{~s}$ of the decay curve has been subtracted. The two dose response curves can be represented by a single saturating exponential growth of the form: $\mathrm{y}=\mathrm{A}\left(1-\exp \left(-\mathrm{D} / \mathrm{D}_{0}\right)\right)$, where $\mathrm{A}$ and $\mathrm{D}_{0}$ are constants. We obtain $\mathrm{D}_{0}$ values of 425 Gy for the initial signal and 280 Gy for the later signal. The natural signals are also shown; the initial natural signal is $\sim 79 \%$ of the saturation level observed in the laboratory, and the later signal is 
$\sim 89 \%$. The measured g-values for these two signals are $2.64 \pm 0.07$ and $2.0 \pm 1.4 \%$ /decade $(n=5)$. This difference in g-values is consistent with the trends in average fading rates for IR(50) presented in Fig. 1. Fig. 2b presents similar information for the $\operatorname{pIRIR}(50,290)$ signal, i.e. the signal stimulated by IR at $290^{\circ} \mathrm{C}$, after an IR exposure at $50^{\circ} \mathrm{C}$. In this case the data are represented by a double saturating exponential with $\mathrm{D}_{0}$ values of 610 and $145 \mathrm{~Gy}$ for the initial signal and 660 and 120 Gy for a signal observed further down the curve (100-140 s, hereafter called 'final'); the initial signal reaches $\sim 97 \%$ of the corresponding laboratory saturation level, and the final signal $\sim 102 \%$. However, despite this apparently stable behaviour, we measure finite g-values for these signals of $1.3 \pm 0.4$ and $0.8 \pm 0.5 \%$ /decade $(\mathrm{n}=6)$, respectively; Thiel et al. (2010) also observed finite laboratory fading rates for their apparently stable signal.

Our data suggest that (i) the observations of Thiel et al. (2010) are applicable to coarse grained K-feldspar

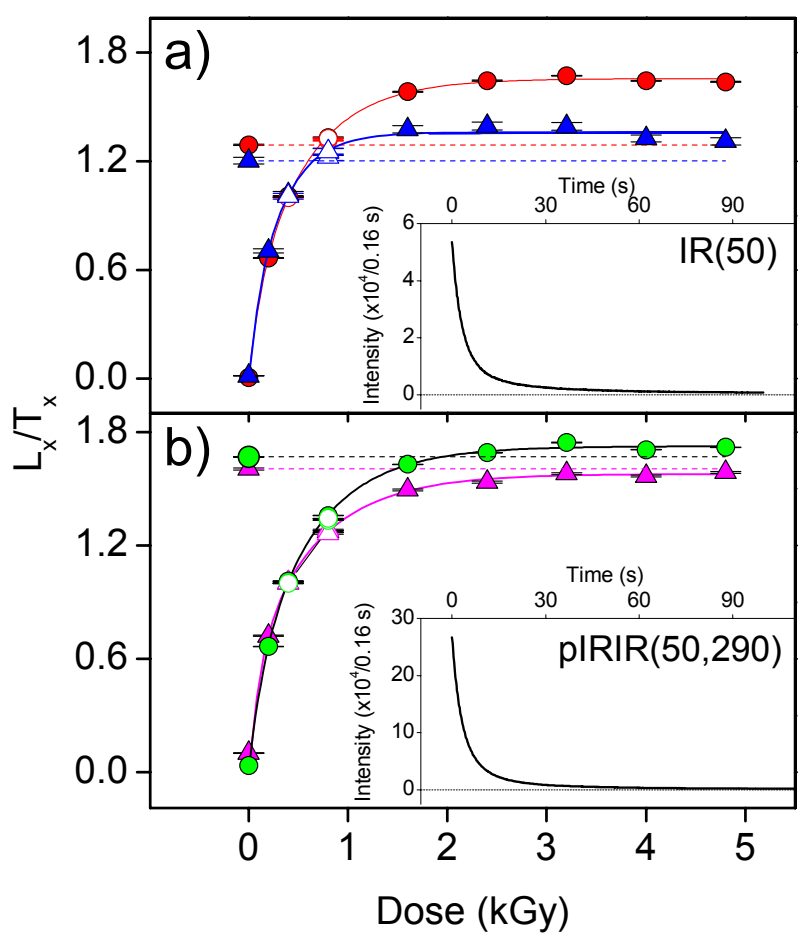

Fig. 2. Sensitivity corrected dose response curves for sample 062213 expected to be in field-saturation using both the initial signal (circles) and the final signal (triangles). Recycling values are shown as open symbols. The sensitivity corrected naturals $\left(L_{n} / T_{n}\right)$ are shown as dashed lines. A preheat of $320^{\circ} \mathrm{C}$ for $60 \mathrm{~s}$ was used. a) Dose response curve for IR stimulation at $50^{\circ} \mathrm{C}(I R(50))$ for $100 \mathrm{~s}$. The initial signal was calculated using the first $1.44 \mathrm{~s}$ of stimulation whereas the final signal was calculated using the signal from 50 to $60 \mathrm{~s}$. The inset shows the decay curve for the first (natural) test dose given a dose of $400 \mathrm{~Gy}$. b) Dose response curve for pIRIR(50,290) stimulation for $400 \mathrm{~s}$. The initial signal was calculated using the first $1.44 \mathrm{~s}$ of stimulation whereas the final signal was calculated using the signal from 100 to $140 \mathrm{~s}$. The inset shows the decay curve of the first (natural) test dose of $400 \mathrm{~Gy}$. extracts, and (ii) our earlier laboratory observations of increasing stability with increasing stimulation temperature, and increasing stability with stimulation time, are applicable to the natural signal. The remainder of this paper uses laboratory experiments to further investigate the origins of the elevated temperature IRSL signal.

\section{PULSE ANNEAL EXPERIMENTS}

We now examine the thermal stability of the signals stimulated by IR and pIRIR for various stimulation times and temperatures to investigate the origins of the IRSL signals.

All the pulse anneal experiments presented here were undertaken using a SAR procedure in which the entire regenerated signal is measured for each preheat temperature. The preheat temperatures ranged from 320 to $650^{\circ} \mathrm{C}$ and were all held for $60 \mathrm{~s}$. After the final $650^{\circ} \mathrm{C}$ measurement, the initial measurement using a preheat of $320^{\circ} \mathrm{C}$ for $60 \mathrm{~s}$ was repeated. Any sensitivity change was monitored using a test dose with a preceding preheat of $320^{\circ} \mathrm{C}$ for $60 \mathrm{~s}$. The regeneration and test doses were both $8 \mathrm{~Gy}$; overall sensitivity change was typically $30-40 \%$, but all recycling values after sensitivity correction are within $10 \%$ of unity. A high-temperature IR stimulation at $330^{\circ} \mathrm{C}$ for $100 \mathrm{~s}$ was inserted after each $\mathrm{L}_{\mathrm{x}}$ and $\mathrm{T}_{\mathrm{x}}$ cycle to minimize recuperation effects.

\section{IRSL measured at $50^{\circ} \mathrm{C}(\operatorname{IR}(50))$}

In this section we examine the thermal stability of the IR(50) signal. Murray et al. (2009) also examined the thermal stability of the IR(50) signal using the first $3.9 \mathrm{~s}$ of stimulation. These authors argued that the main source of the IR(50) signal was a single IR bleachable peak at $\sim 410^{\circ} \mathrm{C}$. Fig. 3 shows the pulse anneal curve for sample 981014 for the initial (first 1.44 s) IR(50) signal (squares). The IR stimulation time in this experiment was $1,000 \mathrm{~s}$. For preheat temperatures $>450^{\circ} \mathrm{C}$, the signal is thermally eroded to $<1 \%$ of its initial value at $320^{\circ} \mathrm{C}$. The shape of the pulse anneal curve is very similar to that presented by Murray et al. (2009).

Thomsen et al. (2008) showed that by using a later part of the decay curve the fading rate is reduced by $\sim 60 \%$ compared to that obtained using the initial signal (see Fig. 1). They explained this change in signal stability in terms of a tunnelling probability exponentially related to the distance between donor-acceptor pairs. In that model, the initial part of the IRSL decay curve would originate with the luminescence emitted from close electron-hole pairs while the latter part represents the tunnelling recombination of more distant pairs. Fig. 3 also presents the pulse anneal curves for later parts of the decay curve as identified in the legend in the inset to Fig. 3. The apparent thermal stability of the signal increases as the signal integration interval is moved further down the decay curve. The same trend is observed for laboratory fading rates, i.e. the fading rates become smaller as the 
signal integration interval is moved further down the decay curve (Fig. 1). It should be noted that the amount of signal remaining after a preheat at $420^{\circ} \mathrm{C}$ increases significantly, from $\sim 5 \%$ (of that at $320^{\circ} \mathrm{C}$ ) for the 0 to $1.44 \mathrm{~s}$ integration interval to $\sim 20 \%$ for the 225 to $300 \mathrm{~s}$ interval.

\section{Elevated temperature IRSL}

Thomsen et al. (2008) also showed that measuring IRSL at elevated temperatures reduced the observed fading rate (see Fig. 1). In Fig. 3 the change in thermal stability for various parts of the decay curve was shown for the IR(50) signal. The inset to Fig. 3 shows the corresponding pulse anneal curves for IR(290). (For comparison both the initial and final (100 to $140 \mathrm{~s}$ ) signals for $\operatorname{IR}(50)$ are also shown as dashed lines.) The IR(290) signal also becomes initially more thermally stable with increasing stimulation time but after $50 \mathrm{~s}$ there is no further change in signal stability. It is also interesting to note that the further down the IRSL decay curve the signal summation is made, the flatter the initial part $\left(<390^{\circ} \mathrm{C}\right)$ of the pulse anneal curve becomes, i.e. the initial signal depletes in a continuous manner whereas the final signal doesn't begin to decrease until the preceding preheat temperature is higher than $390^{\circ} \mathrm{C}$.

The effect of increasing the stimulation temperature on the pulse anneal curve for sample 981014 is shown for the initial signal for stimulation temperatures of 50, 100, 225 and $290^{\circ} \mathrm{C}$ in Fig. 4a (legend given in Fig. 4b); a higher stimulation temperature seems to result in greater thermal stability of the signal. As the thermal stability of this elevated temperature IR signal increases, the laboratory fading rate decreases: the measured g-value of the initial signal is $1.86 \pm 0.13 \% /$ decade $(n=4)$, whereas the

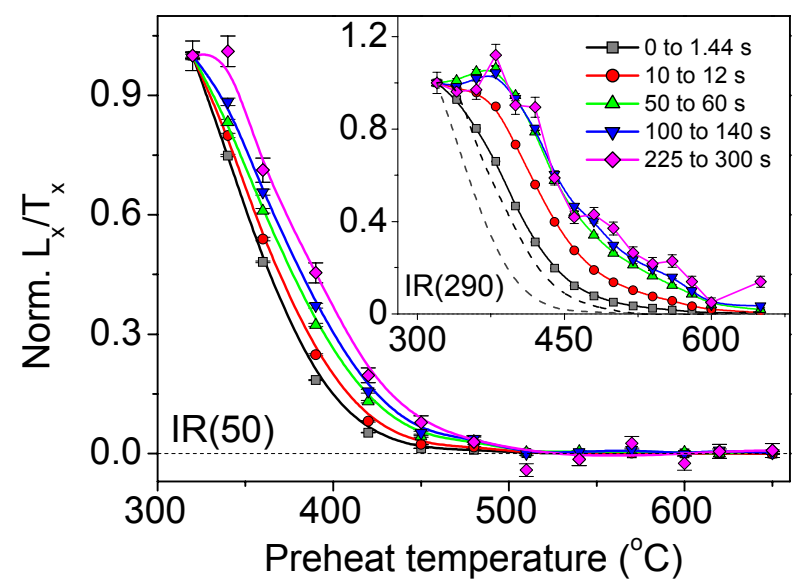

Fig. 3. Pulse anneal curves for $I R(50)$ measured for 1,000 s for different summation intervals down the decay curve of sample 981014 . The data have been normalized to the initial preheat temperature of $320^{\circ} \mathrm{C}$. Legend is given in the inset, which also shows the pulse anneal curves obtained for IR(290) measured for $400 \mathrm{~s}$. The dashed lines in the inset are the pulse anneal curves for the initial and final $I R(50)$ signals. g-value for the final summation interval is $0.94 \pm 0.15 \% /$ decade $(n=4)$.

Thomsen et al. (2008) showed that by stimulating with IR at $50^{\circ} \mathrm{C}$ prior to IR stimulation at elevated temperature (a pIRIR stimulation) a further decrease in the fading rate of the initial signal could be obtained (see Fig. 1). A pulse anneal curve for sample 981014 for the initial pIRIR $(50,290)$ signal $(0-1.44 \mathrm{~s})$ is also shown in Fig. 4a. The prior IR stimulation results in a significantly more thermally stable initial signal than that obtained without prior stimulation. The final signals (100-140 s) for all the stimulation modes presented in Fig. 4a are summarised in Fig. $\mathbf{4 b}$. These signals are all more stable than the corresponding initial signals (Fig. 4a); the $\operatorname{pIRIR}(50,290)$ final signal is also repeated as a dashed line in Fig. 4a for comparison. The pulse anneal curves for stimulation at elevated temperature begin to decrease at higher and higher preheat temperatures as the stimulation temperature is increased, i.e. the higher the stimulation temperature, the larger the preheat temperature that is required for a given thermal depletion. The pulse anneal curves also tend to follow the same trend of increasing stability as the initial signals and the same trend in fading rates (Fig. 1). However, one important difference is that

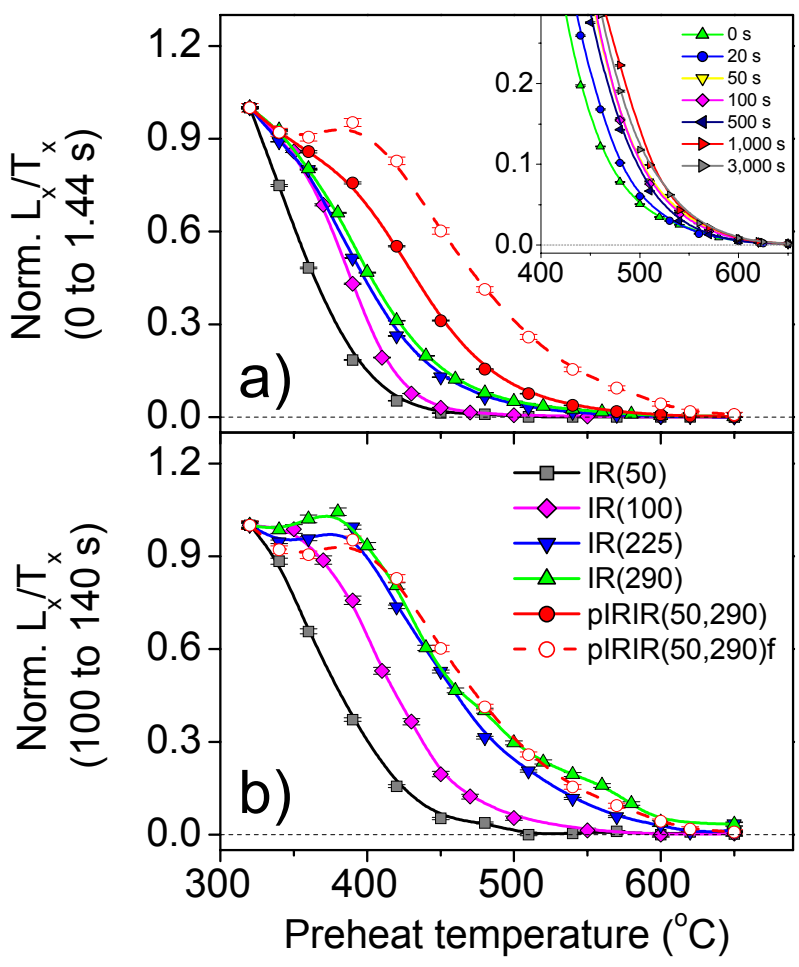

Fig. 4. Pulse anneal curves for sample 981014 for $I R(50), I R(100)$, $I R(225), I R(290)$ and $p \operatorname{IRIR}(50,290)$ for $100 \mathrm{~s}$ of prior $I R(50)$ stimulation. a) Initial signals (first $1.44 \mathrm{~s})$. Also shown is the final signal for pIRIR(50,290) stimulation (pIRIR(50,290)f; dashed line). The legend is given in b). The inset shows the effect of changing the stimulation time of the prior IR stimulation in the pIRIR(50,290) signal. b) Final signal (100-140s). 
the thermal stability of the final IR(225), IR(290) and pIRIR $(50,290)$ signals are all similar; the thermal stability of the final signal is independent of stimulation temperature and prior IR stimulation time for stimulation above $\sim 200^{\circ} \mathrm{C}$. This result is again reflected in the behaviour of the published g-values (Fig. 1), where the difference in the final signals (28.8 to $72.3 \mathrm{~s}$ ) between $\operatorname{IR}(225)$ and $\operatorname{pIRIR}(50,225)$ is negligible.

The pulse anneal curves for sample 981014 show that the IRSL signals measured at elevated temperatures are almost completely thermally depleted by a preheat in the $550^{\circ} \mathrm{C}$ to $650^{\circ} \mathrm{C}$ range (Fig. 4a), whereas the IRSL signal measured at $50^{\circ} \mathrm{C}$ is thermally depleted by a preheat temperature of $\sim 450^{\circ} \mathrm{C}$ (Fig. 4a, squares). The generality of this result was investigated using four other K-feldspar samples (662213, 951020, 963806 and 951012). The pulse anneal curves for the initial $\operatorname{IR}(50)$ and pIRIR(50,290) signals are shown in Fig. 5 (the inset shows a close-up of the data for temperatures from 400 to $650^{\circ} \mathrm{C}$ ). All samples show the same trends as 981014 confirming the generality of our results.

\section{Effect of duration of the prior IR exposure on the pIRIR signal}

In the pIRIR data presented so far, all the prior IR stimulation was for $100 \mathrm{~s}$; it may be that increasing the duration of prior IR stimulation would result in a further increase in thermal stability. This was investigated for the pIRIR(50,290) initial signal (0-1.44 s) using 20, 50, 100, 500,1000 and $3000 \mathrm{~s}$ of prior IR stimulation time; the results are shown inset to Fig. 4a. Increasing the duration of the prior IR stimulation beyond $\sim 100 \mathrm{~s}$ does not seem to result in a significantly more stable pIRIR $(50,290)$

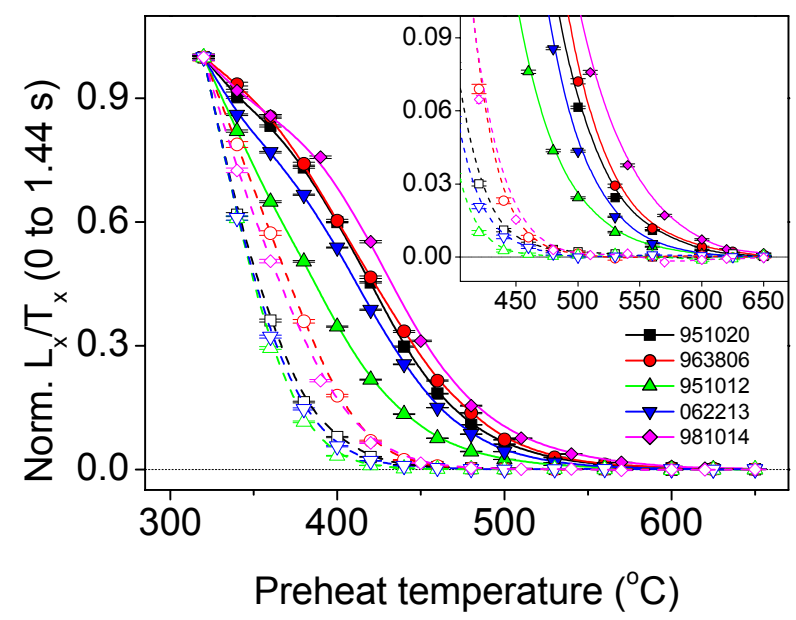

Fig. 5. Pulse anneal curves for pIRIR(50,290) stimulation for samples 951020 (squares), 963806 (circles), 951912 ("upright" triangles), 062213 ("down" triangles) and 981014 (diamonds). Also shown are the pulse anneal curves for IR(50) (open symbols and dashed lines). The inset shows a close up of the data. signal. This is again reflected in the observed change in the corresponding fading rates, where the initial signal gives g-values of $1.86 \pm 0.13(\mathrm{n}=4), 1.00 \pm 0.21 \quad(\mathrm{n}=3)$ and $0.89 \pm 0.10 \% /$ decade $(n=3)$ for $0 \mathrm{~s}, 100 \mathrm{~s}$ and $1000 \mathrm{~s}$ of prior IR stimulation, respectively. For comparison, fading rate measurements using the pIRIR $(50,225)$ signal and a preheat of $250^{\circ} \mathrm{C}$ (rather than $320^{\circ} \mathrm{C}$ as used above) gave g-values of $1.80 \pm 0.03,0.96 \pm 0.11$ and $0.83 \pm 0.19$ and $0.82 \pm 0.08 \% /$ decade $(\mathrm{n}=3)$ for prior IR stimulations of $0,100,1000$ and $3000 \mathrm{~s}$, respectively. Thus, in a pIRIR measurement initial stimulation at $50^{\circ} \mathrm{C}$ for longer than $100 \mathrm{~s}$ does not seem to change either the thermal stability or the laboratory fading rate significantly.

\section{Effect of changing the stimulation temperature on the pIRIR signal}

Thermal transfer in feldspar can be significant especially for young samples (e.g. Huntley and Clague, 1996) and thus it may, in general, be desirable to make use of as low a preheat as possible. The preheat employed should normally be higher than the highest stimulation temperature, so it is useful to identify the lowest possible stimulation temperature that still gives an acceptably stable signal. In Fig. 6a the effect of changing the second stimulation temperature in a pIRIR stimulation is shown for the initial signal for $\operatorname{pIRIR}(50,150)$, $\operatorname{pIRIR}(50,200)$, $\operatorname{pIRIR}(50,225)$ and $\operatorname{pIRIR}(50,290)$. The latter two pIRIR signals have very similar thermal stabilities, whereas the $\operatorname{pIRIR}(50,150)$ and the $\operatorname{PIRIR}(50,200)$ signals are significantly less stable. This suggests that the second IR stimulation temperature should be $>200^{\circ} \mathrm{C}$ to obtain the most thermally stable signal (and thus probably the signal with the least fading). The pulse anneal curves for the final signals are shown in the inset, and they show a similar pattern; there is no difference between the final signals from $\operatorname{pIRIR}(50,225)$ and $\operatorname{pIRIR}(50,290)$.

\section{Effect of changing the stimulation temperature of the prior IR stimulation on the PIRIR signal}

Above it was shown that the duration of the prior IR stimulation at $50^{\circ} \mathrm{C}$ did not significantly affect the signal stability for stimulation times $>100 \mathrm{~s}$. In the following experiments the stimulation time of the prior IR was fixed at $100 \mathrm{~s}$. Figure $6 \mathrm{~b}$ presents pulse anneal curves that result from varying the temperature, $\mathrm{T}$, of the IR stimulation in the pIRIR(T,290) signal, for $\mathrm{T}=50,100,200$ and $250^{\circ} \mathrm{C}$. Increasing the first IR stimulation temperature up to $200^{\circ} \mathrm{C}$ also increases the thermal stability, but the curves for pIRIR $(200,290)$ and $\operatorname{pIRIR}(250,290)$ are indistinguishable. The inset in Fig. $6 \mathbf{b}$ shows the pulse anneal curves based on the final signal. Also shown is the initial pIRIR $(250,290)$ signal. All the curves are indistinguishable from each other; the pIRIR $(250,290)$ signal seems to have the same thermal stability regardless of where the summation interval is placed on the decay curve. 


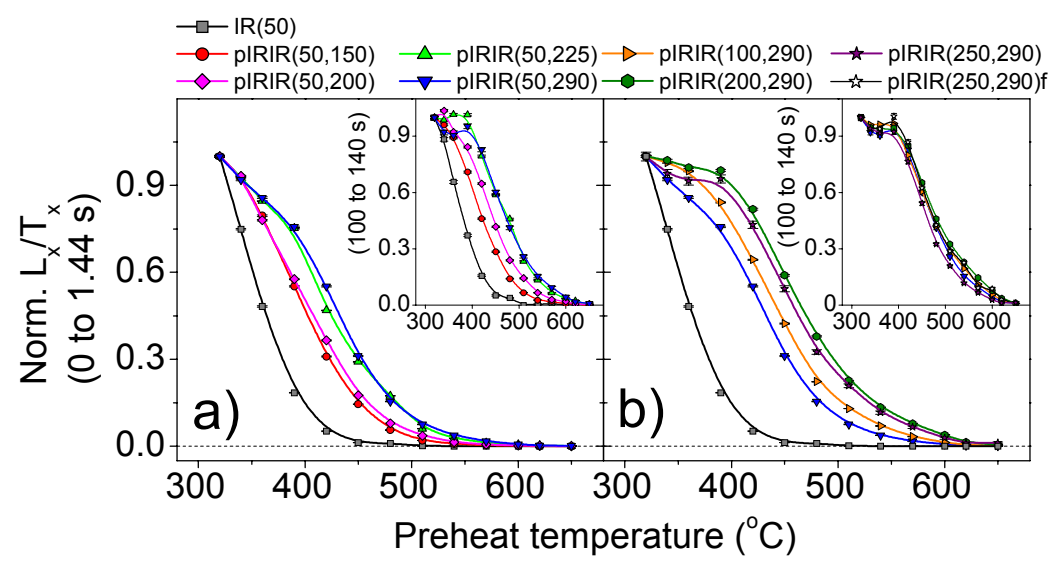

Fig. 6. pIRIR pulse anneal curves for sample 981014 a) Pulse anneal curves for different IR $\left(2^{\text {nd }}\right)$ stimulation temperatures using the initial signal. The $I R(50)$ curve is shown for comparison. The inset shows the curves obtained from the final signal. b) Pulse anneal curves for the initial pIRIR(T,290) signal using a prior $I R$ stimulation of $100 \mathrm{~s}$ at $T=50,100,200$ and $250^{\circ} \mathrm{C}$, respectively. The $I R(50)$ curve is shown for comparison. The inset shows the curves for the final signal. Also shown in the inset is the initial pIRIR(250,290) signal.

\section{Pulse anneal summary}

It is clear that the results of IR stimulation at elevated temperature without prior IR stimulation (Fig. 4) can be explained in terms of a mixture of $\operatorname{IR}(50)$ and pIRIR signals, and the trends in g-values support this. Thus in the following, we focus only on IR(50) and pIRIR signals.

As the summation interval on the IR(50) decay curve is moved progressively to longer stimulation times, the pulse anneal curves (Fig. 3) indicate that the signal becomes more thermally stable. The same pattern is also observed for the pIRIR(50,290) signal (Fig. 4). In both cases these observations follow the observed trend in $\mathrm{g}$ values (see section 3). There is also a weak dependence on thermal stability of the length of the prior IR stimulation for periods of less than $100 \mathrm{~s}$, suggesting that $\sim 100 \mathrm{~s}$ of prior IR(50) stimulation is sufficient to remove the thermally less stable component of the pIRIR signal. Fig. 5 suggests that these observations are not unique to a single sample, but apply at least to the five samples examined here.

Only $\sim 1 \%$ of the IR(50) signal remains for preheats of $\sim 450^{\circ} \mathrm{C}$. The preheat temperature required to reach this level of depletion increases somewhat as the summation interval moves to longer stimulation times. For the initial $\operatorname{pIRIR}(50,290)$ a preheat of $550^{\circ} \mathrm{C}$ is required to reduce this signal by $99 \%$, whereas the final signal requires a preheat of more than $600^{\circ} \mathrm{C}$.

We next examined the dependence of thermal stability on stimulation temperature (Fig. 6). The stability of the pIRIR initial and final signals increases with the temperature of the second stimulation until $\sim 225^{\circ} \mathrm{C}$. At higher temperatures the thermal stabilities are unchanged. The higher the temperature of the first IR stimulation, the greater the thermal stability of the initial part of the pIRIR signal. In contrast, the thermal stability of the later part of the pIRIR signal is independent of the first stimulation temperature.
The simplest interpretation of all these results is that the pIRIR signal is derived from a source which is thermally more stable than that of the $\operatorname{IR}(50)$, i.e. the $\operatorname{IR}(50)$ signal is derived from a trap located somewhat below $450^{\circ} \mathrm{C}$ and the pIRIR signal is at least partly derived from a trap located at $\sim 550^{\circ} \mathrm{C}$. The higher the stimulation temperature and the further down the decay curve the signal summation is made, the greater the contribution from this deeper trap. However, based on the model for luminescence production outlined in section 1 it is also possible to explain the obtained data in terms of the single trap model. In the single trap model the preceding preheat will use up the holes available in near-by recombination sites. IR stimulation at $50^{\circ} \mathrm{C}$ results in the promotion of the trapped electron population to the excited state of the trap from where the electrons may tunnel to close-by recombination sites. If there are holes available for recombination the tunnelling may be accompanied by the emission of light. If there are no available holes, the electrons will not be able to leave the trap. Thus, the fact that the IR(50) signal is almost completely depleted after a preheat of $450^{\circ} \mathrm{C}$ may indicate an almost complete depletion of the available recombination sites rather than a depletion of the electron trap. The second IR stimulation at elevated temperature (in the pIRIR stimulation) will subsequently promote (at least some of) the remaining electrons into the band-tail states from where they can reach more distant recombination centres and so recombine to emit light. Thus, in this view, all the electrons giving rise to IRSL (independent of stimulation temperature) can be derived from a single trap. This effect of prior thermal stimulation in causing nearest neighbour depletion has been shown recently by Time-resolved IRSL and Timeresolved post-IR IRSL data (Jain and Ankjærgaard, 2010). These authors show that a thermally induced decrease in the nearest hole population changes the competition between retrapping and recombination for a given stimulation. Thereafter an increase in the stimulation temperature causes an efficient band tail migration thus allowing recombination with the distance neighbours, thus generating the so called pIRIR signal. 


\section{DECAY CURVE SHAPE}

In quartz the shape of the decay curve contains information on the origin of the OSL signal. Compared to quartz, the mechanisms controlling the feldspar decay curve shape are poorly understood, but it is reasonable to assume that there is similar information about the origin of the IRSL signal from feldspar contained in the shape of the decay curve. This section investigates the influence of preheat temperature and stimulation power on the decay curve shape.

\section{The effect of preheat temperature on the decay shape}

Thomsen et al. (2008) showed that for their samples the decay rate is independent of stimulation temperature, when using a preheat of $250^{\circ} \mathrm{C}$ for $60 \mathrm{~s}$ and UV detection (7.5 mm Hoya U-340). Fig. 7a shows the decay curve shape for the $\operatorname{IR}(50)$ and $\operatorname{pIRIR}(50,290)$ signals after a preheat of $320^{\circ} \mathrm{C}$ for $60 \mathrm{~s}$ detected through a combination of Corning 7-59 and BG 39. Before normalisation to the initial intensity, we subtracted a background integrated over $3 \mathrm{~s}(\operatorname{IR}(50))$ and $15 \mathrm{~s}(\operatorname{pIRIR}(50,290))$ from the respective initial signals. The inset shows the measured signals. The intensity of the $\operatorname{pIRIR}(50,290)$ signal is greater than for the IR(50) signal (as also seen in the insets of Fig. 2). For the same preheat temperature the decay shape of the two signals is very similar.

In the following we examine the effect on the decay shape of changing the preheat temperature. Fig. $7 \mathbf{b}$ shows the sensitivity corrected decay curves for the pIRIR $(50,290)$ signal (please note the logarithmic intensity axis). Increasing the preheat temperature reduces both the initial and the final intensities. It also reduces the decay rate, as illustrated in the inset to Fig. $\mathbf{7 b}$ where the time it takes for the signal to decay to $50 \%$ of its initial value $\left(t_{50}\right)$ is shown as a function of preheat temperature. Also shown are the $t_{50}$ values for IR(50) stimulation. The $\operatorname{IR}(50)$ and pIRIR $(50,290)$ signals have very similar decay rates up to a preheat temperature of $360^{\circ} \mathrm{C}$. Above this temperature the IR(50) signal seems to deplete more slowly than the pIRIR $(50,290)$ signal.

Fig. $7 \mathbf{a}$ and $7 \mathbf{b}$ demonstrate that the shape of the IRSL decay curve is not significantly dependent on stimulation temperature, but does depend on preheat temperature. This observation is consistent with both a single-trap and a multiple-trap source model. In the latter case preheating is simply removing the rapidly decaying, less thermally stable source of electrons leaving behind a more stable source with a lower decay rate. In the case of a singletrap model the preheating is removing the initial part of the decay curve by holding electrons in the excited state and allowing them to tunnel to near-by recombination centres. Thus, in both models the initial part of a subsequent IRSL signal has simply been thermally eroded to a greater extent and thus starts further down the "original" decay curve. This idea has been tested by shifting the decay curves (obtained with a preheat $>320^{\circ} \mathrm{C}$ ) to start at
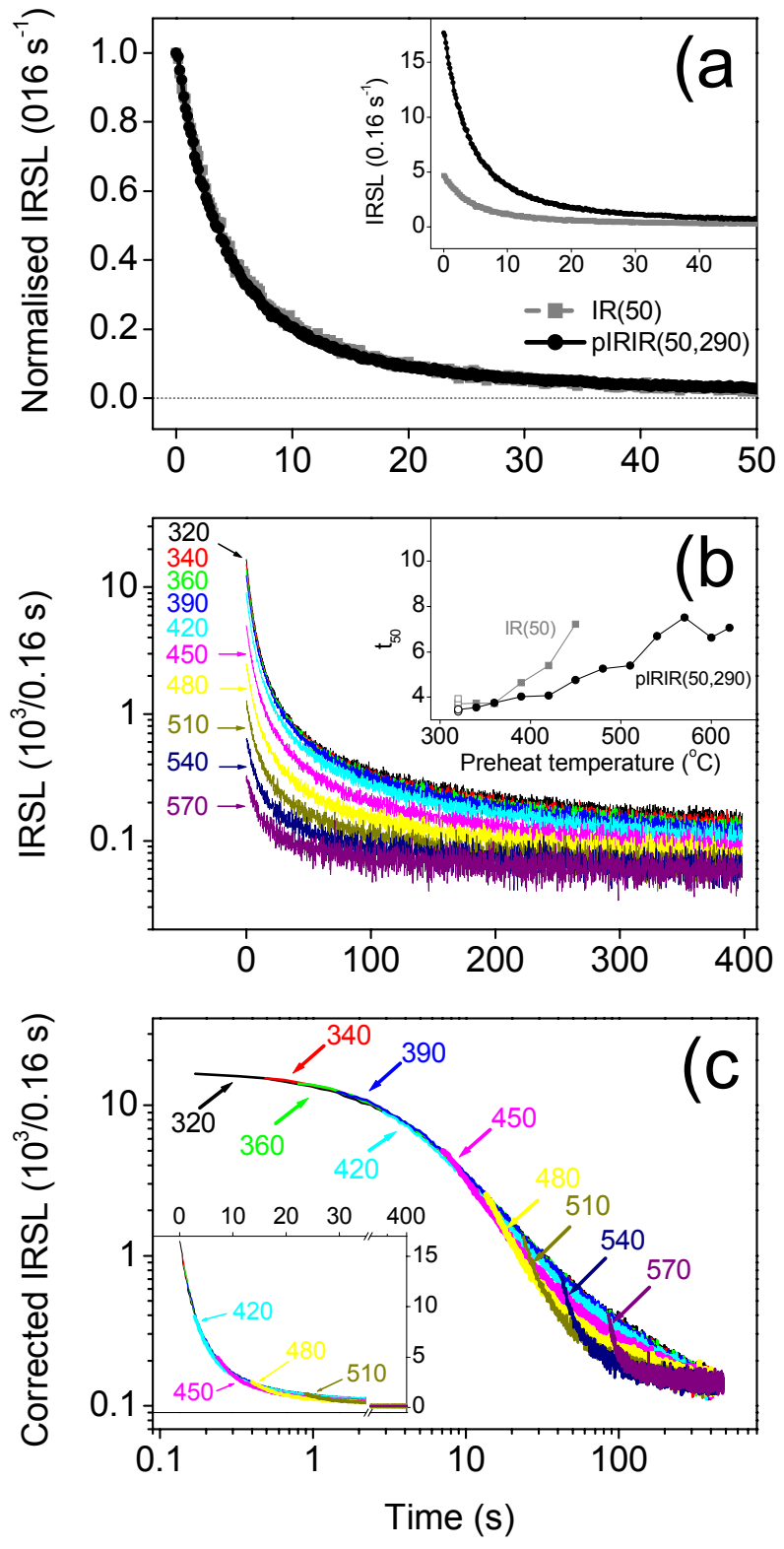

Fig. 7. a) Normalised decay curve shapes for the $I R(50)$ and pIRIR $(50,290)$ signals after a preheat of $320^{\circ} \mathrm{C}$ for $60 \mathrm{~s}$. The inset shows the measured decay curves. b) Decay curves for the sensitivity corrected pIRIR $(50,290)$ signal for various preheat temperatures. The inset shows the time it takes for the $\operatorname{IR}(50)$ and pIRIR $(50,290)$ signals to decay to $50 \%$ of their initial value. c) pIRIR $(50,290)$ decay curves for various preheat temperatures after sensitivity correction and background subtraction. The decay curves have been time shifted so the initial intensity matches that of the decay curve obtained after a preheat of $320^{\circ} \mathrm{C}$. The same data are shown in the inset using a linear axis.

longer stimulation times, so that the initial count rate matches that of the signal obtained using a preheat of $320^{\circ} \mathrm{C}$ (e.g. instead of starting at time $\mathrm{t}=0$, the decay curve is shifted to begin at $10 \mathrm{~s}$ ). Before time shifting the decay curves, a constant was added to ensure that the 
signals reached the same background. Using this approach we confirmed that the $\operatorname{pIRIR}(50,290)$ signals obtained using preheats $<420^{\circ} \mathrm{C}$ have very similar decay rates (Fig. 7c). Above a preheat temperature of $450^{\circ} \mathrm{C}$ the measured signals have significantly different initial decay rates, probably because recuperation (thermal transfer from shallow IR-insenstive traps) becomes more important as the main part of the signal is progressively eroded.

\section{The effect of the IR stimulation power on the decay shape}

We next examine the dependence of feldspar IRSL decay curve shape on stimulation power ranging from $\sim 1.35$ to $\sim 135 \mathrm{~mW} / \mathrm{cm}^{2}$. An aliquot of sample 981014 was given a dose of $7 \mathrm{~Gy}$, preheated at $280^{\circ} \mathrm{C}$ for $60 \mathrm{~s}$ and stimulated with IR at $50^{\circ} \mathrm{C}$ for $10,000 \mathrm{~s}$ using LED power settings ranging between 1 and $100 \%(\sim 1.35$ to $\sim 135 \mathrm{~mW} / \mathrm{cm}^{2}$ ). To monitor sensitivity change a test dose of 7 Gy was measured at a constant power of $90 \%$ $\left(\sim 121 \mathrm{~mW} / \mathrm{cm}^{2}\right)$ for $100 \mathrm{~s}$ between each stimulation. The initial $2 \mathrm{~s}$ of test dose stimulation (less a background of the last $2 \mathrm{~s}$ ) was used to sensitivity correct the individual decay curves (the sensitivity changed by about $25 \%$ over the whole experiment). To ensure that the sensitivity correction was working adequately the stimulation employing a power setting of $80 \%\left(\sim 108 \mathrm{~mW} / \mathrm{cm}^{2}\right)$ was repeated at the end of the experiment; a recycling value of $\sim 0.98$ confirmed that the sensitivity correction was appropriate. A high-temperature stimulation $(90 \%$ power at $280^{\circ} \mathrm{C}$ for $100 \mathrm{~s}$ ) was inserted between each stimulation cycle to minimise recuperation effects. The resulting decay curves (after background subtraction using the last $5 \mathrm{~s}$ of stimulation and sensitivity change correction) are shown in Fig. 8a. In the initial part of the decay curve, the stimulation rate (diode power) has a clear effect on the IRSL signal; the greater the power, the greater is the initial IRSL signal, and the faster the rate of decay of the signal. This confirms that the rate of photon absorption by trapped electrons controls the initial production rate of IRSL and thus presumably the emptying of the IRSL trap(s). However, as the IRSL signal becomes weaker, the decay rate becomes much less dependent on the stimulation rate. For instance, comparing the curves obtained for relative stimulation powers of $100 \%$ and $10 \%$, the intensity and decay rates become very similar after about $30 \mathrm{~s}$ of stimulation. For a stimulation power of only $1 \%$, the decay rate is indistinguishable from those at all higher powers after $\sim 200 \mathrm{~s}$ of stimulation (see Fig. 8a inset). Similar observations were made using the $\operatorname{pIRIR}(50,225)$ signal (data not shown).

These observations are to be expected from the model discussed earlier. For close electron/hole pairs, the probability for tunnelling from the excited state is high and this leads to very rapid recombination. In these circumstances, the initial IRSL signal and initial decay rate will be governed by the rate of stimulating charge into the excited state. As close pairs are used up, the tunnelling
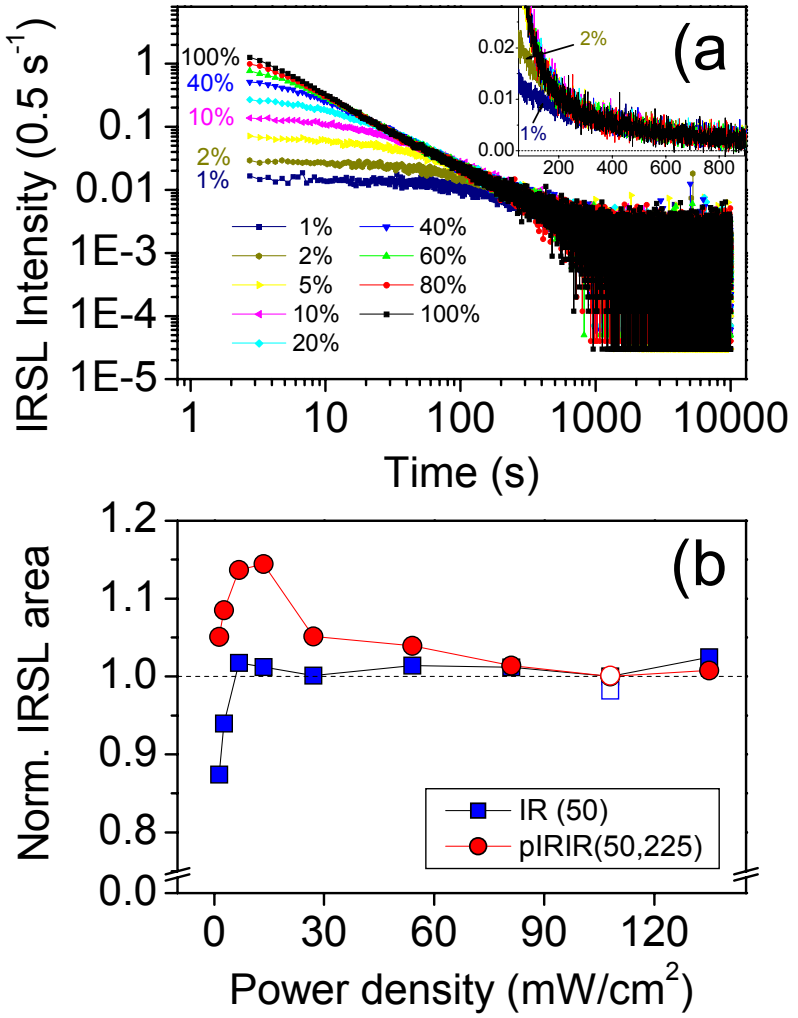

Fig. 8. a) Effect of changing the stimulation power on decay shape for the $I R(50)$ signal from sample 981014 . A background using the last $5 \mathrm{~s}$ of stimulation has been subtracted and the curves have been corrected for sensitivity change (see text for details). The inset shows a close up of the data. b) The area of the individual decay curves for a constant number of incident photons as a function of incident power for the $\operatorname{IR}(50)$ and $\operatorname{pIRIR}(50,225)$ signals.

rate decreases until it begins to control the rate of luminescence production. The longer the stimulation, the further apart are the remaining pairs, and so even for very low stimulation rates, the tunnelling rate controls luminescence production and the excitation rate ceases to have a significant influence.

In Fig. 8b the area under the IRSL decay curves for a constant number of incident photons (equivalent to $100 \mathrm{~s}$ of stimulation at $100 \%$ power) is shown as a function of stimulation power for $\operatorname{IR}(50)$ and $\operatorname{pIRIR}(50,225)$ stimulation. The curve obtained using a diode power setting of $100 \%\left(\sim 135 \mathrm{~mW} / \mathrm{cm}^{2}\right)$ was summed for the initial $100 \mathrm{~s}$, while the curve using $10 \%\left(\sim 13.5 \mathrm{~mW} / \mathrm{cm}^{2}\right)$ was summed for the initial $1,000 \mathrm{~s}$. For power settings greater than $5 \%$ $\left(\sim 6.8 \mathrm{~mW} / \mathrm{cm}^{2}\right)$ the number of detected photons per stimulation photon is constant for the IR(50) signal. For lower power settings the number of detected photons drops to $\sim 87 \%$. This drop may be an artefact of the instrumentation - it is not known whether the diode power is accurate at very low power settings. For pIRIR $(50,225)$ stimulation, below a power setting of $30 \%$ $\left(\sim 40.5 \mathrm{~mW} / \mathrm{cm}^{2}\right)$ the IRSL area for the same number of 
incident photons appear to become more efficient; i.e. the IRSL production is more efficient for the pIRIR signal that it is for the $\operatorname{IR}(50)$ signal, although this integrated signal also drops at $<5 \%$ of full power.

\section{POSSIBLE SOURCE OF THE ELEVATED TEM- PERATURE IRSL SIGNAL}

Murray et al. (2009) concluded that the main source of the IRSL signal measured at $50^{\circ} \mathrm{C}$ is an IR bleachable TL peak at $\sim 410^{\circ} \mathrm{C}$. The data for the IR(50) signal presented in this paper are consistent with this conclusion. However, the pulse anneal experiments of the elevated temperature IRSL signal (Fig. $\mathbf{4 b}$ ) may suggest that this signal originates from a deeper trap at $\sim 550^{\circ} \mathrm{C}$. To examine whether the elevated temperature IRSL signal originates from a deeper trap than the IRSL measured at $50^{\circ} \mathrm{C}$, a series of high temperature thermoluminescence (TL) experiments were performed. An aliquot of sample 981014 was first heated to $700^{\circ} \mathrm{C}$ to erase any residual IR signal, and then given a dose of $1.7 \mathrm{kGy}^{1}$, preheated to $450^{\circ} \mathrm{C}$ for $60 \mathrm{~s}$ and subsequently held at $290^{\circ} \mathrm{C}$ for $1,000 \mathrm{~s}$ before $\mathrm{TL}$ measurement to $700^{\circ} \mathrm{C}$ at $1{ }^{\circ} \mathrm{C} / \mathrm{s}$ (Fig. 9a). The experiment was then repeated, but this time the sample was stimulated with IR for $1,000 \mathrm{~s}$ while being held at $290^{\circ} \mathrm{C}$ before the final TL measurement to $700^{\circ} \mathrm{C}$. The peak at $550^{\circ} \mathrm{C}$ is detectably reduced by this prior IR stimulation, although the effect is small. Finally, the experiment was repeated one more time, with IR simulation for $1,000 \mathrm{~s}$ at $50^{\circ} \mathrm{C}$ before the final TL measurement. The peak at $550^{\circ} \mathrm{C}$ appears to be unaffected by this low temperature prior IR stimulation. The inset to Fig. 8a summarises the loss of TL as a result of IR stimulation. Stimulation at $50^{\circ} \mathrm{C}$ has no detectable effect on the TL peak, whereas IR stimulation at $290^{\circ} \mathrm{C}$ results in a significant reduction on the lower temperature side of the TL peak, and also gives some phototransfer at $\sim 420^{\circ} \mathrm{C}$.

The observed loss in TL could be caused by IR depletion of the source trap; if so one would expect the loss of TL to be proportional to the area of the IRSL decay curve. To investigate the relationship between the loss of TL and IRSL area the following experiment was conducted using a dose of $\sim 650 \mathrm{~Gy}$ :

1) Dose - PH(450) for $60 \mathrm{~s}-$ Hold at temperature (T) for $320 \mathrm{~s}-\mathrm{TL}(700)$

2) Dose - PH(450) for $60 \mathrm{~s}-\mathrm{IR}(\mathrm{T})$ for $\mathrm{x} \mathrm{s}-$ Hold at temperature (T) for (320-x) s - TL(700)

3) Dose $-\mathrm{PH}(450)$ for $60 \mathrm{~s}-$ Hold at temperature (T) for $320 \mathrm{~s}-\mathrm{TL}(700)$

The duration of IR stimulation, $\mathrm{x}$, was $0,5,10,20,40$, 80160 and $320 \mathrm{~s}$, respectively. Steps 1 and 3 were used

\footnotetext{
${ }^{1}$ Measuring TL at high temperatures using blue detection filters is difficult because of black body radiation. In these experiments the black body signal was reduced by inserting a card board filter with a single pin hole in the blue detection filter pack. However, this also reduces the luminescence intensity that we are measuring, so a high dose was given to the sample to ensure measurement of a detectable signal.
}

to monitor and correct for any sensitivity change taking place throughout the experiment. Fig. 9b shows the loss of TL caused by prior IR stimulation at temperature $\mathrm{T}\left(50,150\right.$ and $290^{\circ} \mathrm{C}$, respectively) as a function of IRSL area (loss of TL calculated using the TL area from 450 to $650^{\circ} \mathrm{C}$ ). The relationship between apparent loss of TL and IRSL area is consistent with a straight line passing through the origin, indicating that the elevated temperature IRSL signal could be derived from this TL trap. However, Murray et al. (2009) demonstrated that the loss in area of a TL peak at $\sim 140^{\circ} \mathrm{C}$ was proportional to the IRSL signal but showed, nevertheless, that it did not contribute significantly to the IRSL production; rather, the loss of TL was explained in terms of changes in luminescence recombination probability. Thus, if we assume that the IRSL and the TL make use of the same recombination centres, we cannot rule out the possibility that the
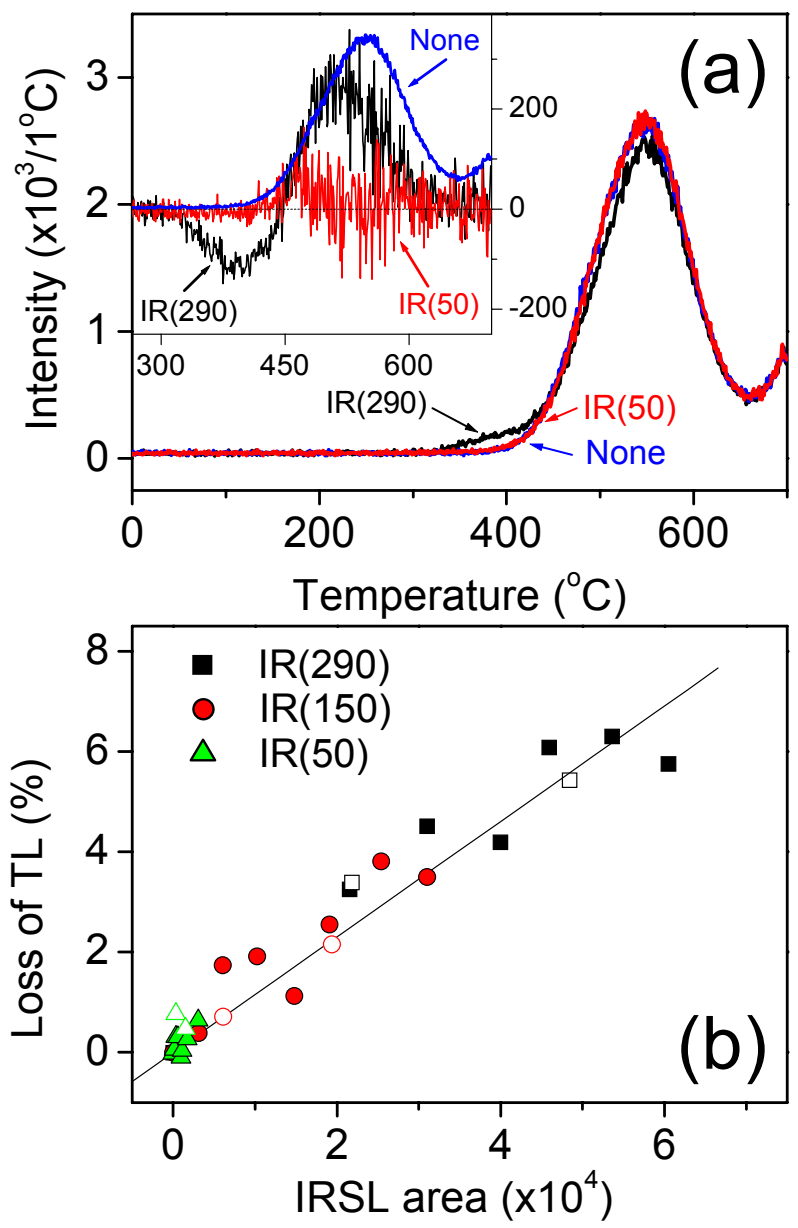

Fig. 9. a) The effect of prior IR stimulation for $1,000 \mathrm{~s}$ at $50^{\circ} \mathrm{C}$ and $290^{\circ} \mathrm{C}$ on the $T L$ curve. Also shown is the $T L$ curve with no prior IR stimulation (None, see text for details). The inset shows the loss of $T L$. Also shown is a scaled version of the TL curve with no prior IR stimulation. b) Loss of $T L$ from 450 to $650^{\circ} \mathrm{C}$ as a function of total IRSL area for stimulation temperatures of $50^{\circ} \mathrm{C}$ (triangles), $150^{\circ} \mathrm{C}$ (circles) and $290^{\circ} \mathrm{C}$ (squares). Open symbols are recycling points. 
observed loss of TL is caused simply by the using up of available holes by the prior IR stimulation and not by trap depletion.

\section{DOSE RESPONSE CURVES}

One of the attractive features of using feldspar as a natural dosimeter is that the dose response curve has been reported to grow to more than $1 \mathrm{kGy}$ using the IRSL signal measured at $50^{\circ} \mathrm{C}$. Thus, it is important to establish whether the elevated temperature IRSL signals investigated here also grow to large doses. This was examined by measuring dose response curves using a single aliquot of sample $981014 \mathrm{KF}$ for various IRSL signals. Regeneration doses ranging from 20 to 3000 Gy where employed in a SAR protocol. A test dose of 180 Gy was inserted to monitor and correct for sensitivity changes. Two recycling doses at 108 and 432 Gy were used. All recycling values were within $10 \%$ of unity. The preheat temperature (held for $60 \mathrm{~s}$ ) was the same for both regeneration and test dose measurements. The resulting dose response curves (see Fig. 10) were fitted with a single or a double saturating exponential as required and the resulting $\mathrm{D}_{0}$ values are given in Table 1 (useful dose estimates are usually derived for natural doses $\leq 2 \times \mathrm{D}_{0}$ ). Using a preheat of $275^{\circ} \mathrm{C}$ for $60 \mathrm{~s}$ four different dose response curves were measured for $\operatorname{IR}(50), \operatorname{IR}(245)$ and $\operatorname{pIRIR}(50,245)$ for 100 and $1,000 \mathrm{~s}$ of prior IR(50) stimulation. All the initial signals were fitted with a single saturating exponential and the $\mathrm{D}_{0}$ values are $1042 \pm 11$, $990 \pm 20,769 \pm 18$ and $746 \pm 17 \mathrm{~Gy}$, respectively. Thus, for this preheat temperature the initial $\mathrm{D}_{0}$ values are $\sim 25 \%$ smaller for the pIRIR signal than for the IR(50) signal. As expected the $\mathrm{D}_{0}$ values for the $\operatorname{pIRIR}(50,245)$ signal with a prior IR(50) stimulation of 100 and $1,000 \mathrm{~s}$ are indistinguishable from each other. The dose response curves constructed using the final signals have all been fitted using a double saturating exponential. If the final signal is used the larger $D_{0}$ values $\left(D_{0, a}\right.$ in Table 1$)$ from the various signals are indistinguishable from one another and the average $D_{0}$ value is $903 \pm 24$ Gy. The average smaller $D_{0}$ value $\left(D_{0, b}\right.$ in Table 1$)$ is $208 \pm 31 \mathrm{~Gy}$.

From the pulse anneal data the IR(50) signal is almost completely removed by a preheat of $450^{\circ} \mathrm{C}$ (e.g. Fig. 3).
When using pIRIR stimulation we attempt to remove this signal optically, but one could also attempt to remove it thermally by preheating to $450^{\circ} \mathrm{C}$. We investigated the effect of increasing the preheat temperature to $450^{\circ} \mathrm{C}$ on the $\mathrm{D}_{0}$ value for the $\operatorname{IR}(50)$, IR(245) signal and the pIRIR $(50,245)$ signal with a prior IR stimulation time of $100 \mathrm{~s}$. With a preheat of $450^{\circ} \mathrm{C}$ we did not expect to see a significant difference between the $\mathrm{D}_{0}$ values of the latter two signals, since the high preheat temperature ought to have eliminated the need for the IR(50) stimulation. Both the initial and final signal required a double saturating exponential and the $\mathrm{D}_{0}$ values for the initial signals are slightly lower than those determined using a preheat temperature of $275^{\circ} \mathrm{C}$. The $\mathrm{D}_{0, \mathrm{a}}$ values from the final signal are significantly smaller, especially for the

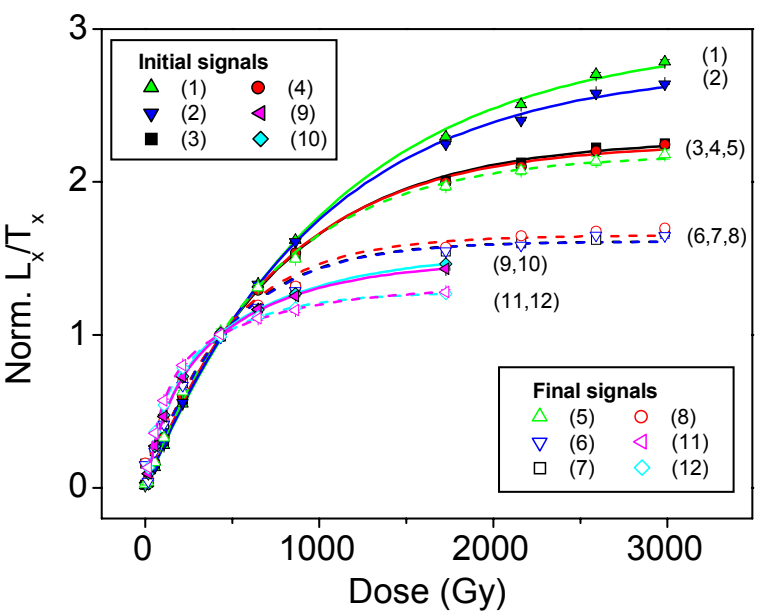

Fig. 10. Dose response curves for sample 981014 for various stimulation $\left(50\right.$ and $\left.245^{\circ} \mathrm{C}\right)$ and preheat temperatures $\left(275\right.$ and $\left.450^{\circ} \mathrm{C}\right)$. The dose response curves have been normalised to unity at the second recycling point (432 Gy). Dose response curves constructed using the initial signal are given as filled symbols and solid lines and the dose response curves for the final signal as open symbols and dashed lines. Dose response curves (1) to (8) all used a preheat of $275^{\circ} \mathrm{C}$ for $60 \mathrm{~s}$, whereas (9) to (12) made use of a preheat of $450^{\circ} \mathrm{C}$ for $60 \mathrm{~s}$. IR(50): (1),(5); IR(245): (2),(6),(9),(11); pIRIR(50,245) with $100 \mathrm{~s}$ of prior IR stimulation: (3),(7); pIRIR(50,245) with $1,000 \mathrm{~s}$ of prior IR stimulation: (4), (8), (10),(12).

Table 1. $D_{0}$ values for sample 981014 for stimulation temperatures of 50 and $245^{\circ} \mathrm{C}$ for both initial and final signals. Two different preheat temperatures $(\mathrm{PH})$ of $275^{\circ} \mathrm{C}, 60 \mathrm{~s}$ and $450^{\circ} \mathrm{C}, 60 \mathrm{~s}$ were used. No. refers to the dose response curve number given in Fig. 10. pIRIR $t$ refers pIRIR(50,245) stimulation with a prior IR stimulation time of $t(t=100$ or 1,000$)$.

\begin{tabular}{|c|c|c|c|c|c|c|c|}
\hline \multirow{2}{*}{$\mathrm{PH}\left({ }^{\circ} \mathrm{C}\right)$} & \multirow{2}{*}{ No. } & \multirow{2}{*}{ Stimulation } & \multicolumn{2}{|c|}{$\begin{array}{l}\text { Initial signal } \\
\end{array}$} & \multirow{2}{*}{ No. } & \multicolumn{2}{|c|}{ Final signal } \\
\hline & & & $\mathrm{D}_{0, \mathrm{a}}(\mathrm{Gy})$ & $\mathrm{D}_{0, \mathrm{~b}}(\mathrm{~Gy})$ & & $\mathrm{D}_{0, \mathrm{a}}(\mathrm{Gy})$ & $\mathrm{D}_{0, \mathrm{~b}}(\mathrm{~Gy})$ \\
\hline \multirow{4}{*}{275} & 1 & $\mathrm{IR}(50)$ & $1042 \pm 11$ & - & 5 & $971 \pm 170$ & $299 \pm 110$ \\
\hline & 2 & $\mathrm{IR}(245)$ & $990 \pm 20$ & - & 6 & $885 \pm 180$ & $184 \pm 36$ \\
\hline & 3 & pIRIR 100 & $769 \pm 18$ & - & 7 & $893 \pm 183$ & $188 \pm 35$ \\
\hline & 4 & pIRIR 1,000 & $746 \pm 17$ & - & 8 & $862 \pm 134$ & $162 \pm 31$ \\
\hline \multirow{2}{*}{450} & 9 & $I R(245)$ & $570 \pm 54$ & $114 \pm 15$ & 11 & $585 \pm 48$ & $94 \pm 5$ \\
\hline & 10 & pIRIR 100 & $677 \pm 59$ & $127 \pm 10$ & 12 & $408 \pm 46$ & $69 \pm 12$ \\
\hline
\end{tabular}


$\operatorname{pIRIR}(50,245)$ measurement. It is interesting to note that the shape of the dose response curves using a preheat of $450^{\circ} \mathrm{C}$ (dose response curves 9 to 12 in Fig. 10) is significantly different from that using a preheat of $275^{\circ} \mathrm{C}$. This implies that attempting to remove the IR(50) signal thermally results in a different signal from that obtained after optical removal. These data may indicate the presence of at least two traps, a relatively shallow trap which can be stimulated by IR at any temperature and a deeper trap which is only accessed at elevated temperatures. For preheat temperatures less than $450^{\circ} \mathrm{C}$ the initial signal is completely dominated by the shallow trap, but as the stimulation progresses the deeper trap begins to play a role.

It appears from these data that the more stable signal(s) in this sample saturate more quickly than the less stable IR(50) signal, and that, with one exception (pIRIR $(50,245)$ with a preheat of $\left.450^{\circ} \mathrm{C}\right)$, the final and initial signals for any given treatment all have similar $\mathrm{D}_{0}$ values. From a dosimetric perspective, all the $\mathrm{D}_{0}$ values from the various approaches to measurement of the more stable signal are still much larger than those typical for quartz (Wintle and Murray, 2006), and the advantages of using this more stable feldspar signal are still significant.

\section{SUMMARY AND CONCLUSION}

From Figs. 1 and 2 we conclude that pIRIR signals are more stable than $\operatorname{IR}(50)$ both in nature and in the laboratory. In particular there is evidence that the natural $\operatorname{pIRIR}(50,290)$ signal is able to reach saturation on laboratory dose response curve, i.e. it does not show detectable signal loss in nature although a low fading rate is observed on laboratory time scales.

From the pulse anneal curves presented in Figs. 3, 4 and 6 we conclude that the IR(50) and pIRIR signals have different thermal stabilities. This difference in thermal stability is mirrored in laboratory measured fading rates (g-values). Similar thermal stability of the pIRIR signals for a number of $\mathrm{K}$-feldspar separates from sedimentary samples is demonstrated in Fig. 5.

Fig. 7 demonstrates that the shape of the IRSL decay curve is not significantly dependent on stimulation temperature, but does depend on preheat temperature. This observation is consistent with the idea of both a singletrap and a multiple-trap source. In the latter case preheating is simply removing the rapidly decaying, less thermally stable source leaving behind a more stable source with a lower fading loss. In the case of a single-trap model the preheating is removing the initial part of the decay curve by holding electrons in the excited state and allowing them to recombine with near-by recombination centres.

Although the shape of the IRSL decay curve does not change significantly the initial intensity increases with stimulation temperature (see Fig. 2 insets and Fig. 7a inset). This observation is consistent with the idea that only a small fraction of the total trapped electron population is able to tunnel to near-by recombination centres during low temperature stimulation (e.g. Trautmann et al., 2000), but a larger fraction (giving more luminescence) if the stimulation temperature is increased.

In Fig. 8 we show that the rate of photon absorption by trapped electrons controls the initial production rate of IRSL and thus presumably the emptying of the IRSL $\operatorname{trap}(\mathrm{s})$. However, at longer stimulation times the decay rate is independent of the rate of photon absorption, indicating that the tunneling rate controls the IRSL production. We further show that for IR(50) the efficiency of the luminescence production is constant as a function of the rate of IR stimulation, but for pIRIR the stimulation at lower rates appears to be more efficient at producing luminescence than for the IR(50) stimulation.

In Fig. 9 we show the existence of a high temperature TL peak centred on $\sim 550^{\circ} \mathrm{C}$, some small fraction of which is bleachable by IR light at elevated temperatures (but not at $50^{\circ} \mathrm{C}$ ); this bleachable component seems to peak somewhat below $550^{\circ} \mathrm{C}$. Presumably the high temperature TL peak is actually made up of two peaks, only the smaller of which is IR sensitive. The observed loss in TL for prior IR(150) and IR(290) stimulation is proportional to the IRSL area, which could indicate that a least a part of the IR signal measured at an elevated temperature originates with the trap(s) giving rise to the IR sensitive TL peak. However, the observed data could also be explained by a single trap model, for two reasons: (i) the trap may still be the same as the IR(50) trap, in that it may have the same trap depth etc. Such a single trap could give rise to a TL peak skewed to high temperatures - the lower temperature component arising from tunnelling from the excited state, as discussed above, and the higher temperature tail from the extra thermal energy required to excite charge into higher energy band-tail states (or possibly into the conduction band) so that it can access more distant recombination centres (unavailable for tunnelling because of the trap/centre spacing); (ii) the apparent loss of charge from the higher temperature TL peak may result from a loss of holes in recombination centres (and so a reduction in luminescence recombination probability); in this model the holes are used up by the IR stimulated charge, but there is no need for this charge to be derived from the TL peak under consideration.

Although we cannot make unambiguous statements about the origins of the more stable elevated temperature IRSL signal, it is still important to establish how the various IRSL signals grow with dose and in particular to determine the $\mathrm{D}_{0}$ values. In Fig. 10 and Table 1 we show that the more stable signals saturate more quickly than the less stable signals and that the initial and final signals saturate at approximately the same level.

In conclusion it appears that a stable IRSL signal can be measured from sand-sized grains of K-feldspar; this signal is certainly more stable (both thermally and in 
terms of tunnelling probability) than the conventional IRSL signal measured at $50^{\circ} \mathrm{C}$, and indeed this signal may reach dose saturation before instability becomes detectable. For the sample examined here, the $\operatorname{pIRIR}(50,245)$ signal (both initial and final) has a $\mathrm{D}_{0}$ value of $\sim 800 \mathrm{~Gy}$, which suggests that a natural dose of $\sim 1600$ Gy could be determined; this in turn implies an age limit of $\sim 500-600 \mathrm{ka}$ for sedimentary coarse grain K-feldspars.

\section{REFERENCES}

Buylaert JP, Murray AS and Huot S, 2008. Optical dating of an Eemian site in Northern Russia using K-feldspar. Radiation Measurements 43: 715-720, DOI 10.1016/j.radmeas.2008.01.027.

Buylaert JP, Murray AS and Thomsen KJ, 2009. Testing the potential of an elevated temperature IRSL signal from K-feldspar. Radiation Measurements 44: 560-565, DOI 10.1016/j.radmeas.2009.02.007.

Buylaert JP, Huot S, Murray AS and Van den haute P, 2010. Infrared stimulated luminescence dating of an Eemian (MIS 5e) site in Denmark using K-feldspar. Boreas (In press), DOI 10.1111/j.1502-3885.2010.00156.x.

Bøtter-Jensen L, Andersen CE, Duller GAT and Murray AS, 2003. Developments in radiation, stimulation and observation facilities in luminescence measurements. Radiation Measurements 37: 535541, DOI 10.1016/S1350-4487(03)00020-9.

Huntley DJ and Lamothe M, 2001. Ubiquity of anomalous fading in Kfeldspars and the measurement and correction for it in optical dating. Canadian Journal of Earth Sciences 38: 1093-1106, DOI 10.1139/cjes-38-7-1093

Huntley DJ and Clague JJ, 1996. Optical dating of tsunami-laid sands. Quaternary Research 46: 127-140.

Jain M and Ankjærgaard C, 2010. Towards finding a non-fading signal in feldspar: insight into charge transport and tunnelling from timeresolved optically stimulated luminescence. Radiation Measurements (accepted).

Lamothe M, Auclair M, Hamazaoui C and Huot S, 2003. Towards a prediction of long-term anomalous fading of feldspar IRSL. Radiation Measurements 37: 493-498, DOI 10.1016/S13504487(03)00016-7.

Murray AS, Buylaert JP, Thomsen KJ and Jain M, 2009. The effect of preheating on the IRSL signal from feldspar. Radiation Measurements 44: 554-559, DOI 10.1016/j.radmeas.2009.02.004.

Poolton NRJ, Bøtter-Jensen L, Ypma PJM and Johnsen O, 1994. Influ- ence of crystal structure on the optically stimulated luminescence properties of feldspars. Radiation Measurements 23: 551-554, DOI 10.1016/1350-4487(94)90098-1.

Poolton NRJ, Bøtter-Jensen L and Johnsen O, 1995. Influence on donor electron energies of the chemical composition of $\mathrm{K}, \mathrm{Na}$ and $\mathrm{Ca}$ aluminosilicates. Journal of Physics: Condensed Matter 7: 47514762, DOI 10.1016/1350-4487(94)00114-G.

Poolton NRJ, Ozanyan KB, Wallinga J, Murray AS and Bøtter-Jensen L, 2002a. Electrons in feldspar II: a consideration of the influence of conduction band-tail states on luminescence processes. Physics and Chemistry Minerals 29: 217-225, DOI 10.1007/s00269-001$0218-2$.

Poolton NRJ, Wallinga J, Murray AS, Bulur E and Bøtter-Jensen L, $2002 \mathrm{~b}$. Electrons in feldspar I: on the wavefunction of electrons trapped at simple lattice defects. Physics and Chemistry Minerals 29: 210-216, DOI 10.1007/s00269-001-0217-3.

Ramos A and Cunha PP, 2004. Facies associations and palaeogeography of the Zanclean-Piacenzian marine incursion in the Mondego Cape Nazaré area (onshore of central Portugal). 23rd IAS Meeting of Sedimentology, Coimbra, September 15-17, 2004, Abstracts Book: 227.

Silva CM, Landau B, Domènech R and Martinell J, 2010. Pliocene Atlantic molluscan assemblages from the Mondego Basin (Portugal): Age and palaeoceanographic implications. Palaeogeography, Palaeoclimatology, Palaeoecology 285: 248-254, DOI 10.1016/j.palaeo.2009.11.016

Spooner NA, 1994. The anomalous fading of infrared-stimulated luminescence from feldspars. Radiation Measurements 23: 625-632, DOI 10.1016/1350-4487(94)90111-2.

Thiel C, Buylaert JP, Murray AS, Terhost B, Hofer I, Tsukamoto S and Frechen M, 2010. Luminescence dating of the Stratzing loess profile (Austria) - Testing the potential of an elevated temperature post-IR IRSL protocol (Accepted in Quaternary International)

Thomsen KJ, Murray AS, Jain M and Bøtter-Jensen L, 2008. Laboratory fading rates of various luminescence signals from feldsparrich sediment extracts. Radiation Measurements 43: 1474-1486, DOI 10.1016/j.radmeas.2008.06.002.

Trautmann T, Krbetschek MR, Dietrich A and Stolz W, 2000. The basic principle of radioluminescence dating and a localized transition model. Radiation Measurements 32: 487-492, DOI 10.1016/S1350-4487(00)00119-0.

Wintle AG, 1973. Anomalous fading of thermoluminescence in mineral samples. Nature 245: 143-144, DOI 10.1038/245143a0.

Wintle AG and Murray AS, 2006. A review of quartz optically stimulated luminescence characteristics and their relevance in singlealiquot regeneration dating protocols. Radiation Measurements 41: 369-391, DOI 10.1016/j.radmeas.2005.11.001. 